\title{
A 44-year-old man with acute asymmetric polyarthritis and fever
}

\author{
Jeffrey Yu MD, Sameer Elsayed MD, Dongmei Sun MD MSc
}

Cite as: CMAJ 2017 June 26;189:E861-4. doi: 10.1503/cmaj.170093

A 44-year-old man presented to hospital with painful swelling of his ankles and right knee, hand, wrist and shoulder. Two weeks earlier, he had experienced acute malaise, headache, myalgias, subjective fevers, vomiting and diarrhea, which eventually resolved. He did not have sore throat, eye pain, back pain or genitourinary symptoms.

He had a history of mild psoriasis, bilateral ankle fractures, right metacarpal fracture and right knee anterior cruciate ligament repair. He took no medications, drank little alcohol and denied smoking or intravenous drug use.

On physical examination, his temperature was $37.9^{\circ} \mathrm{C}$. There was swelling, erythema and severe tenderness over his right hand and forearm (Figure $1 \mathrm{~A}$ ). His ankles were tender and a large right knee effusion was present (Figure 1B). There were no rashes except for long-standing psoriatic plaques over his knees and elbows. Dactylitis, nail pitting, onycholysis, bony deformities and enthesitis were absent. Vertebral range of motion was normal and there was no sacroiliac joint tenderness. The rest of his examination was unremarkable.

Investigations showed a leukocyte count of 10.3 (normal $4-10) \times 10^{9} / \mathrm{L}$ and C-reactive protein (CRP) of $340(0-5) \mathrm{mg} / \mathrm{L}$. Two sets of blood cultures were drawn. Plain radiographs of the affected joints showed old posttraumatic changes. Chest x-ray was normal.

\section{What should be included in your differential diagnosis?}

a. Septic arthritis

b. Seronegative inflammatory arthritis (spondyloarthritis), including reactive arthritis

c. Rheumatoid arthritis or other systemic rheumatic diseases

d. Crystal-related arthritis

e. Sarcoidosis

A review outlines an approach to this differential diagnosis. ${ }^{1}$ Septic arthritis (a) and seronegative inflammatory arthritis (b) should be included in the differential diagnosis.

Sarcoidosis can present as acute arthritis. The triad of bilateral lower limb arthritis, hilar lymphadenopathy and erythema nodosum is diagnostic of sarcoidosis without requiring further investigations, and is known as Löfgren syndrome. It is often selflimited. ${ }^{1}$ Our patient had a normal chest $\mathrm{x}$-ray and did not have erythema nodosum.

Crystal arthropathies such as gout or pseudogout can present acutely in a mono- or polyarticular distribution, but systemic symptoms such as profound malaise and fever are less common. ${ }^{1}$

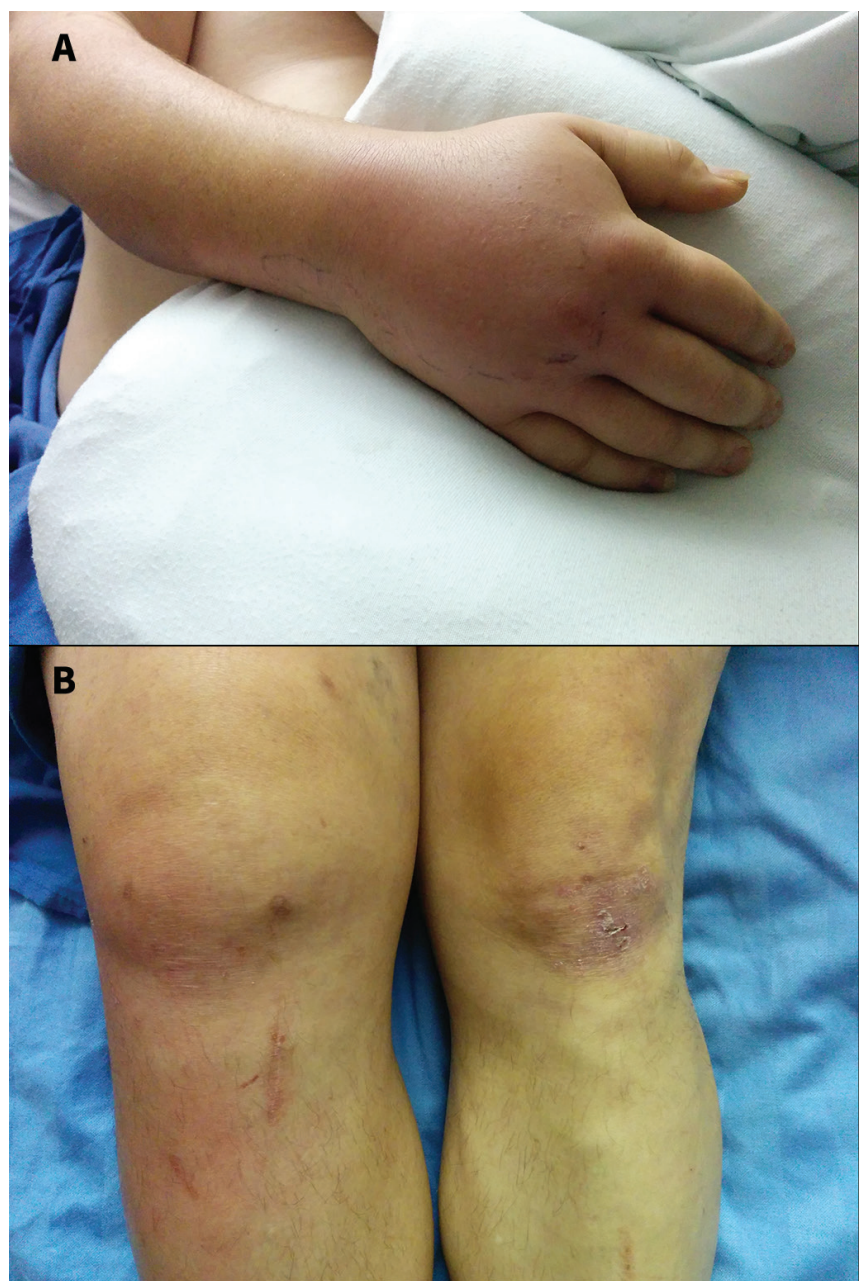

Figure 1: (A) Tender, warm, edematous and erythematous right hand, suggestive of cellulitis, with possible underlying joint effusion, of a 44-year-old man. (B) Right knee effusion post-aspiration. Scars are present from previous anterior cruciate ligament repair. Psoriatic plaques are present on the left knee. 
Rheumatoid arthritis usually develops symmetrically with morning stiffness in smaller joints. Other rheumatic diseases, such as systemic lupus erythematosus, typically have other associated clinical or laboratory features. ${ }^{1}$

A seronegative spondyloarthritis, (b), is high on the differential. Psoriatic arthritis can develop regardless of skin disease severity. Reactive arthritis can follow acute gastrointestinal infections. Ankylosing spondylosis or enteropathic arthritis can be considered, although our patient did not have any back pain or chronic diarrhea. ${ }^{1}$

Septic arthritis, (a), is also high on the differential for acute arthritis with joint effusions and fever. Viral infectious arthritis is often polyarticular, but arthritis from bacterial infections, such as Staphylococcus aureus and Streptococcus species, is usually monoarticular. Disseminated gonococcal infection is another common cause. As described in a systematic review, septic arthritis remains the most important diagnostic consideration, because delayed treatment for bacterial infections can result in substantial morbidity or mortality. ${ }^{2}$ Because bacterial septic arthritis develops through hematogenous spread, predisposing factors must be explored: intravenous drug abuse; immunocompromised states, including HIV; damaged or prosthetic joints; and infective endocarditis.

\section{After blood cultures are drawn, what is the most appropriate next step?}

a. Nonsteroidal anti-inflammatory drugs (NSAIDs), while waiting for results of rheumatoid factor, anticyclic citrullinated peptide, and antinuclear antibodies testing

b. Magnetic resonance imaging (MRI) of affected joints

c. Empiric intravenous antibiotics

d. Joint aspiration, then empiric intravenous antibiotics

e. Empiric corticosteroids

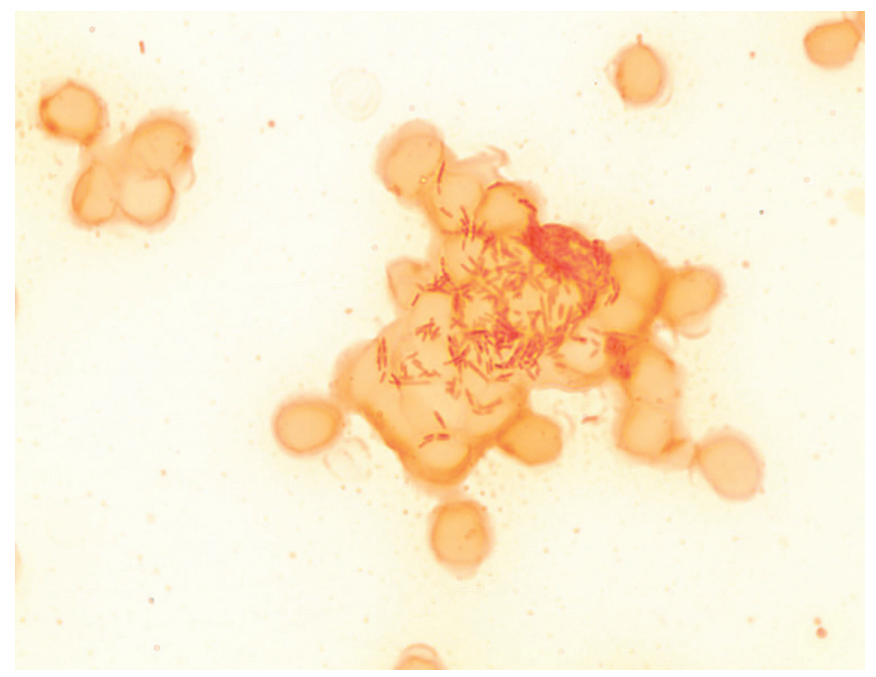

Figure 2: Gram stain from synovial fluid, after culture bottles were flagged positive. Image taken at original magnification x 1000 under oil immersion. There are pleomorphic, filamentous, gram-negative rods arranged in chains and tangles, characteristic of Streptobacillus moniliformis.
The most appropriate next step is joint aspiration (d). Although NSAIDs may provide symptomatic relief and serologies help to explore the differential diagnosis, infection must be ruled out immediately. Empiric corticosteroids are inappropriate when infection is suspected. MRI may assist in detecting osteomyelitis, but is unhelpful in diagnosing septic arthritis.

Our patient's fever and systemic symptoms raised the suspicion of septic arthritis. A systematic review describes joint aspiration as the best diagnostic tool for detecting an infected synovium. ${ }^{2}$ Elevated synovial white blood cell count and polymorphonuclear cell percentage should prompt empiric antibiotic coverage while awaiting culture results, although abnormal cell counts can be seen in noninfectious inflammatory arthritides. Starting antibiotics before synovial fluid sampling may result in a false-negative culture.

We did not attempt joint aspiration on the patient's right wrist, owing to overlying cellulitis. Instead, his large right knee effusion was aspirated, which yielded cloudy yellow fluid, $49.8 \times$ $10^{9} / \mathrm{L}$ nucleated cells, with $88 \%$ polymorphonuclear neutrophils. Crystal analysis and initial Gram stain were negative.

The patient was admitted and started on intravenous cefazolin. On post-admission day 2, his knee aspirate grew Streptobacillus moniliformis (Figure 2). Subsequent history-taking revealed that the patient regularly purchased live rats to feed pet snakes. He denied any bites or scratches. Two days before onset of his systemic symptoms, he had used his bare hands to clean up snake and rat feces.

The patient's antibiotic was changed to intravenous penicillin $G$ 2 million units every four hours for polyarticular streptobacillary septic arthritis. Blood cultures were negative throughout his hospital stay. Transthoracic echocardiogram did not show any signs of endocarditis. The orthopedic surgery service recommended against immediate operative joint drainage; however, this was to be re-evaluated if the patient failed a full course of medical therapy. He continued to improve clinically and his CRP normalized. Four days later, he was discharged to complete a two-week course of intravenous penicillin $\mathrm{G}$. At completion of the course of intravenous penicillin $\mathrm{G}$, he still complained of mild wrist pain, and treatment was extended to two additional weeks of intravenous ceftriaxone $2 \mathrm{~g}$ daily. He was asymptomatic after four weeks of intravenous antibiotics. He ended up giving his pet snakes away.

\section{Discussion}

Streptobacillus moniliformis is a gram-negative facultative anaerobe that colonizes the nasopharyngeal flora of healthy rats, as noted in a systematic review. ${ }^{3}$ Human infection occurs in about $10 \%$ of rat bites, but rat-bite fever may be a misnomer, as many cases may not be associated with an actual bite. Transmission may also occur through through exposure to rat secretions or feces. There have been more than 200 documented cases of $S$. moniliformis infection in the United States, with additional reports in Canada, Mexico, South America, Europe and Australia. Only nine cases of confirmed S. moniliformis infection have been documented in Canada since the 1950s. ${ }^{4-10}$ 


\section{Clinical presentation}

Classic streptobacillary rat-bite fever is a systemic febrile illness with asymmetric migratory polyarthralgias (Box 1 ). The arthralgias may progress to an aseptic joint inflammation (reactive arthritis). If untreated, the mortality rate for rat-bite fever is about $10 \%,{ }^{3}$ as the infection can lead to sepsis or end-organ invasion such as endocarditis. There is no estimate on the prevalence of positive blood cultures, as growth or identification of S. moniliformis is challenging.

Less commonly, S. moniliformis septic arthritis can occur. A review of streptobacillary septic arthritis suggests this may be a syndrome distinct from classic rat-bite fever (Box 2). ${ }^{11}$ Blood cultures are usually negative. Polyarticular involvement is common, and synovial fluid typically shows active synovitis with cultures positive for S. moniliformis. Joint abnormalities such as osteoarthritis or previous trauma are postulated to increase the risk of $S$. moniliformis seeding. ${ }^{11}$

Our patient did not have a rash or bacteremia, and his clinical course closely followed the nonclassic streptobacillary septic polyarthritis syndrome. However, we did not aspirate other joints to truly confirm polyarticular infection.

\section{Diagnosis}

Rat-bite fever is a laboratory diagnosis (Figure 2). Because of its nonspecific clinical features and the frequent absence of a rat bite, a high degree of clinical suspicion must be communicated to the laboratory, as specific expertise is needed to isolate S. moniliformis in appropriate media. ${ }^{3}$

Noninflammatory polyarthralgias, aseptic reactive polyarthritis or septic polyarthritis may arise. When joint effusion is present,

Box 1: Clinical syndrome of classic streptobacillary ratbite fever ${ }^{3}$

- Fevers and rigors are cardinal symptoms at three to seven days after exposure.

- Associated nonspecific flu-like illness: headache, malaise, nausea and vomiting, myalgias, sore throat.

- Fifty percent of patients subsequently develop asymmetric migratory polyarthralgias that may persist for months to years, or progress to an aseptic synovitis (reactive arthritis).

- Seventy-five percent of patients develop a rash in peripheral extremities: maculopapular, petechial, purpuric or hemorrhagic vesicular.

\section{Box 2: Clinical syndrome of streptobacillary septic} arthritis $^{11}$

- Presenting complaint is usually polyarticular joint pain and swelling that affects knees, ankles or wrists, with evidence of active synovitis (septic polyarthritis).

- Fifty-eight percent of patients have febrile systemic symptoms.

- Only $17 \%$ of patients develop the typical rash seen in classic rat-bite fever. septic arthritis can be diagnosed by aspiration of synovial fluid showing elevated nucleated cells, high percentage of polymorphonuclear leukocytes and positive culture. ${ }^{2}$

Possible complications such as endocarditis, pericarditis, myocarditis, vasculitis, pneumonia or other infectious seeding should be ruled out. ${ }^{3}$

\section{Management}

Streptobacillus moniliformis is readily susceptible to a wide range of antibiotics. In case studies and reviews, intravenous penicillin $\mathrm{G}$ is well described to be curative. ${ }^{3}$ Successful doses range from 400000 to 24 million international units divided daily, depending on disease severity or complications such as septic arthritis or endocarditis. Alternative successful intravenous regimens include nafcillin or ampicillin, cephalosporins, tetracyclines or vancomycin. Intravenous penicillins and cephalosporins are used most often as initial therapy, followed by step-down to an oral equivalent when the patient improves. ${ }^{11}$

The appropriate duration of antibiotic therapy remains unclear. Most cases of uncomplicated rat-bite fever have been successfully treated with a 14-day course. However, in case reviews for streptobacillary septic arthritis, duration ranged from 10 days to six weeks, with a median of four weeks. ${ }^{11,12}$ For infective endocarditis, a six-week regimen is usually chosen.

The role of early surgical management in S. moniliformis septic arthritis is undefined. In a series of 12 septic arthritis cases that were all successfully treated, only three had surgical drainage. ${ }^{11}$

\section{Conclusion}

Polyarthritis is commonly encountered in clinical practice. Careful history-taking and physical examination, in concert with selective laboratory and imaging tests, can lead to the correct diagnosis. It is important that in the presence of acute systemic symptoms, fever and joint effusion, aspiration be performed to rule out an infection.

Streptobacillus moniliformis is an uncommon but important cause of acute polyarticular disease. The classic syndrome of febrile illness, rash and arthralgias or aseptic reactive arthritis is well described in the literature. However, a seemingly distinct entity of polyarticular septic arthritis is becoming more recognized. Both can be readily treated by antibiotics such as intravenous penicillin, but the presence of septic arthritis likely requires a longer duration of treatment and possibly surgical intervention. This again highlights the importance of having a low threshold to perform diagnostic joint aspiration. Future studies are needed to determine the optimal regimen and duration of antibiotic treatment.

\section{References}

1. Meador R, Schumacher $\mathrm{H}$. Evaluating and treating patients with polyarthritis of recent onset. Hosp Physician 2003;39:37-45.

2. Margaretten ME, Kohlwes J, Moore D, et al. Does this adult patient have septic arthritis? JAMA 2007;297:1478-88.

3. Elliott SP. Rat bite fever and Streptobacillus moniliformis. Clin Microbiol Rev 2007;20:13-22.

4. Abdulaziz $\mathrm{H}$, Touchie $\mathrm{C}$, Toye $\mathrm{B}$, et al. Haverhill fever with spine involvement. $J$ Rheumatol 2006;33:1409-10. 
5. Albedwawi S. A teenager with fever, rash and arthritis [published erratum in CMAJ 2006;175:624]. CMAJ 2006;175:354.

6. Dolman CE, Kerr D, Chang H, et al. Two cases of rat-bite fever due to Streptobacillus moniliformis. Can J Public Health 1951;42:228-41.

7. Holden FA, Mackay J. Rat-bite fever - an occupational hazard. Can Med Assoc J 1964;91:78-81.

8. Khatchadourian $\mathrm{K}$, Ovetchkine $\mathrm{P}$, Minodier $\mathrm{P}$, et al. The rise of the rats: a growing pediatric issue. Paediatr Child Health 2010;15:131-4.

9. McKee G, Pewarchuk J. Rat-bite fever. CMAJ 2013;185:1346.

10. Schachter ME, Wilcox L, Rau N, et al. Rat-bite fever, Canada. Emerg Infect Dis 2006;12:1301-2.

11. Wang TK, Wong S. Streptobacillus moniliformis septic arthritis: a clinical entity distinct from rat-bite fever? BMC Infect Dis 2007;7:56.

12. Dendle C, Woolley I, Korman T. Rat-bite fever septic arthritis: illustrative case and literature review. Eur J Clin Microbiol Infect Dis 2006;25:791-7.
Competing interests: None declared.

This article has been peer reviewed.

The authors have obtained patient consent.

Affiliations: Department of Medicine (Yu, Elsayed, Sun); Department of Pathology and Laboratory Medicine (Elsayed), Schulich School of Medicine and Dentistry, Western University, London, Ont.

Contributors: Jeffrey Yu drafted the manuscript. All authors provided patient care in the case and made revisions to the manuscript for important intellectual content. All authors approved the final version for publication and agreed to act as guarantors of the work.

Correspondence to: Jeffrey Yu, jeffrey.yu@lhsc.on.ca 